\title{
Rewriting of Regular Expressions and Regular Path Queries
}

\author{
Diego Calvanese, Giuseppe De Giacomo, Maurizio Lenzerini \\ Dipartimento di Informatica e Sistemistica, Università di Roma "La Sapienza," \\ Via Salaria 113, I-00198 Roma, Italy
}

E-mail: calvanese@dis.uniroma1.it, degiacomo@dis.uniroma1.it, lenzerini@dis.uniroma1.it

and

Moshe Y. Vardi

Department of Computer Science, Rice University, P.O. Box 1892, Houston, Texas 77251-1892

E-mail: vardi@cs.rice.edu

Received September 7, 1999; revised June 19, 2001; published online March 6, 2002

Recent work on semi-structured data has revitalized the interest in path queries, i.e., queries that ask for all pairs of objects in the database that are connected by a path conforming to a certain specification, in particular to a regular expression. Also, in semi-structured data, as well as in data integration, data warehousing, and query optimization, the problem of view-based query rewriting is receiving much attention: Given a query and a collection of views, generate a new query which uses the views and provides the answer to the original one. In this paper we address the problem of view-based query rewriting in the context of semi-structured data. We present a method for computing the rewriting of a regular expression $E$ in terms of other regular expressions. The method computes the exact rewriting (the one that defines the same regular language as $E$ ) if it exists, or the rewriting that defines the maximal language contained in the one defined by $E$, otherwise. We present a complexity analysis of both the problem and the method, showing that the latter is essentially optimal. Finally, we illustrate how to exploit the method for view-based rewriting of regular path queries in semi-structured data. The complexity results established for the rewriting of regular expressions apply also to the case of regular path queries. 2002 Elsevier Science (USA)

Key Words: semistructured data; query rewriting; regular path queries; regular expressions; computational complexity.

\section{INTRODUCTION}

Database research has often shown strong interest in path queries, i.e., queries that ask for all pairs of objects in the database that are connected by a specified 
path (see for example [CMW87, CM90]). Recent work on semi-structured data has revitalized such interest. Semi-structured data are data whose structure is irregular, partially known, or subject to frequent changes [Abi97]. They are usually formalized in terms of labeled graphs, and capture data as found in many application areas, such as web information systems, digital libraries, and data integration [BDFS97, CACS94, MMM97, QRS + 95].

The basic querying mechanism over such graphs is the one that retrieves all pairs of nodes connected by a path conforming to a given pattern. Since a user may ignore the precise structure of the graph, the mechanism for specifying path patterns should be flexible enough to allow for expressing regular path queries, i.e., queries that provide the specification of the requested paths through a regular language $\left[\mathrm{AQM}^{+}\right.$97, BDHS96, $\mathrm{FFK}^{+98}$ ]. For example, the regular path query $\left({ }^{*} \cdot(\right.$ rome + jerusalem $) \cdot{ }^{*} \cdot$ restaurant $)$ specifies all the paths having at some point an edge labeled rome or jerusalem, followed by any number of other edges and by an edge labeled with a restaurant.

Methods for reasoning about regular path queries have been recently proposed in the literature. In particular, [AV97, BFW98] investigate the decidability of the implication problem for path constraints, which are integrity constraints that are exploited in the optimization of regular path queries. Also, containment of conjunctions of regular path queries has been addressed and proved decidable in [CDGL98, FLS98].

In semi-structured data, as well as in data integration, data warehousing, and query optimization, the problem of view-based query rewriting is receiving much attention [U1197, Hal00, DGL00]: Given a query $Q$ and $k$ queries $Q_{1}, \ldots, Q_{k}$ associated with the symbols $q_{1}, \ldots, q_{k}$, respectively, generate a new query $Q^{\prime}$ over the alphabet $q_{1}, \ldots, q_{k}$ such that, first interpreting each $q_{i}$ as the result of $Q_{i}$, and then evaluating $Q^{\prime}$ on the basis of this interpretation, provides the answer to $Q$.

Several papers investigate this problem for the case of conjunctive queries (with or without arithmetic comparisons) [LMSS95, RSU95], queries with aggregates [SDJL96, CNS99], recursive queries [DG97], disjunctive views [DG98, AGK99], nonrecursive queries and views for semi-structured data [PV99], and queries expressed in Description Logics [BLR97]. Rewriting techniques for query optimization are described, for example, in [CKPS95, ACPS96, TSI96], and in [FS98, MS99] for the case of path queries in semi-structured data. For some relevant prior work see [Con71].

None of the above papers provides a method for rewriting regular path queries. Observe that such a method requires a technique for the rewriting of regular expressions, i.e., the problem that, given a regular expression $E_{0}$, and other $k$ regular expressions $E_{1}, \ldots, E_{k}$, checks whether we can re-express $E_{0}$ by a suitable combination of $E_{1}, \ldots, E_{k}$. As noted in [MS99], such a problem is still open.

In this paper we present the following contributions:

- We describe a method for computing the rewriting of a regular expression $E_{0}$ in terms of other regular expressions. The method computes the exact rewriting (the one that defines the same regular language as $E_{0}$ ) if it exists, or the rewriting that defines the maximal language contained in the one defined by $E_{0}$, otherwise. 
- We provide a complexity analysis of the problem of rewriting regular expressions. We show that our method computes the rewriting in 2EXPTIME, and is able to check whether the computed rewriting is exact in 2EXPSPACE. We also show that the problem of checking whether there is a nonempty rewriting is EXPSPACE-complete, and demonstrate that our method for computing the rewriting is essentially optimal. Finally, we show that the problem of verifying the existence of an exact rewriting is 2EXPSPACE-complete.

- We illustrate how to exploit the above mentioned method in order to devise an algorithm for the rewriting of regular path queries for semi-structured databases. The complexity results established for the rewriting of regular expressions apply to the new algorithm as well. Also, we show how to adapt the method in order to compute rewritings with specific properties. In particular, we consider partial rewritings (which are rewritings that, besides $E_{1}, \ldots, E_{k}$, may use also symbols in $\left.E_{0}\right)$, in the case where an exact one does not exist.

We point out that the results established in this work provide the first decidability results for rewriting recursive queries using recursive views. Indeed, in our context, both the query and the views may contain a form of recursion due to the presence of transitive closure. Observe that the case where the query contains unrestricted recursion has been shown undecidable, even when the views are not recursive [DG97]. More precisely, the authors in [DG97] present a method that computes the maximally contained rewriting of a Datalog query in terms of a set of conjunctive queries, and show that it is undecidable to check whether the rewriting is equivalent to the original query.

The paper is organized as follows. Section 2 presents the method for rewriting regular expressions. Section 3 describes the complexity analysis of both the method and the problem. Section 4 illustrates the use of the technique to rewrite path queries for semi-structured databases. Finally, Section 5 describes possible developments of our research.

\section{REWRITING OF REGULAR EXPRESSIONS}

In this section, we present a technique for the following problem: Given a regular expression $E_{0}$ and a finite set $\mathscr{E}=\left\{E_{1}, \ldots, E_{k}\right\}$ of regular expressions over an alphabet $\Sigma$, re-express, if possible, $E_{0}$ by a suitable combination of $E_{1}, \ldots, E_{k}$.

We assume that associated with $\mathscr{E}$ we always have an alphabet $\Sigma_{\mathscr{E}}$ containing exactly one symbol for each regular expression in $\mathscr{E}$, and we denote the regular expression associated with the symbol $e \in \Sigma_{\mathscr{E}}$ with $r e(e)$. Given any language $\ell$ over $\Sigma_{\mathscr{E}}$, we denote by $\exp _{\Sigma}(\ell)$ the expansion of $\ell$ wrt $\mathscr{E}$, i.e., the language over $\Sigma$ defined as follows

$$
\exp _{\Sigma}(\ell)=\bigcup_{e_{1} \cdots e_{n} \in \ell}\left\{w_{1} \cdots w_{n} \mid w_{i} \in L\left(\operatorname{re}\left(e_{i}\right)\right)\right\}
$$

where $L(e)$ is the language defined by the regular expression $e$. Thus, $\exp _{\Sigma}(\ell)$ denotes all the words obtained from a word $e_{1} \cdots e_{n} \in \ell$ by substituting for each $e_{i}$ 
all words of the regular language associated with $e_{i}$. Given a $\Sigma_{\mathscr{E}}$-word $w$, $\exp _{\Sigma}(\{w\})$ is simply called the expansion of $w$.

DEFINITION 2.1. Let $R$ be any formalism for defining a language $L(R)$ over $\Sigma_{\mathscr{E}}$. We say that $R$ is a rewriting of $E_{0}$ wrt $\mathscr{E}$ if $\exp _{\Sigma}(L(R)) \subseteq L\left(E_{0}\right)$.

Note that we do not constrain in any way the form of the rewritings, which, a priori, need not even be recursive. We are interested in maximal rewritings, i.e., rewritings that capture in the best possible way the language defined by the original regular expression $E_{0}$.

Definition 2.2. A rewriting $R$ of $E_{0}$ wrt $\mathscr{E}$ is $\Sigma$-maximal if for each rewriting $R^{\prime}$ of $E_{0}$ wrt $\mathscr{E}$ we have that $\exp _{\Sigma}\left(L\left(R^{\prime}\right)\right) \subseteq \exp _{\Sigma}(L(R))$. A rewriting $R$ of $E_{0}$ wrt $\mathscr{E}$ is $\Sigma_{\mathscr{E}}$-maximal if for each rewriting $R^{\prime}$ of $E_{0}$ wrt $\mathscr{E}$ we have that $L\left(R^{\prime}\right) \subseteq L(R)$.

Intuitively, when considering $\Sigma$-maximal rewritings we look at the languages obtained after substituting each symbol in the rewriting by the corresponding regular expression over $\Sigma$, whereas when considering $\Sigma_{\mathscr{E}}$-maximal rewritings we look at the languages over $\Sigma_{\mathscr{E}}$. Observe that by definition all $\Sigma$-maximal rewritings define the same language (similarly for $\Sigma_{\mathscr{E}}$-maximal rewritings), and that not all $\Sigma$-maximal rewritings are $\Sigma_{\mathscr{E}}$-maximal, as shown by the following example.

EXAMPLE 2.1. Let $E_{0}=a^{*}, \mathscr{E}=\left\{a^{*}\right\}$, and $\Sigma_{\mathscr{E}}=\{e\}$, where $r e(e)=a^{*}$. Then both $R_{1}=e^{*}$ and $R_{2}=e$ are $\Sigma$-maximal rewritings of $E_{0}$ wrt $\mathscr{E}$, but $R_{1}$ is also $\Sigma_{\mathscr{E}}$-maximal while $R_{2}$ is not.

However, it turns out that $\Sigma_{\mathscr{E}}$-maximality is a sufficient condition for $\Sigma$-maximality.

THEOREM 2.1. Let $R$ be a rewriting of $E_{0}$ wrt $\mathscr{E}$. If $R$ is $\Sigma_{\mathscr{E}}$-maximal then it is also ¿-maximal.

Proof. Assume by contradiction that $R$ is a $\Sigma_{\mathscr{E}}$-maximal rewriting of $E_{0}$ wrt $\mathscr{E}$ that is not $\Sigma$-maximal. Then there is a rewriting $R^{\prime}$ of $E_{0}$ wrt $\mathscr{E}$, a $\Sigma_{\mathscr{E}}$-word $u^{\prime} \in L\left(R^{\prime}\right)$, and a $\Sigma$-word $w \in L\left(\exp _{\Sigma}\left(\left\{u^{\prime}\right\}\right)\right)$ such that for no $\Sigma_{\mathscr{E}}$-word $u \in L(R)$, it holds that $w \in L\left(\exp _{\Sigma}(\{u\})\right)$. Hence $u^{\prime} \notin L(R)$ and $L\left(R^{\prime}\right) \nsubseteq L(R)$. Contradiction.

Given $E_{0}$ and $\mathscr{E}$, we are interested in deriving a $\Sigma$-maximal rewriting of $E_{0}$ wrt $\mathscr{E}$. We show that such a maximal rewriting always exists (although it may be empty). In fact, we provide a method that, given $E_{0}$ and $\mathscr{E}$, constructs a $\Sigma_{\mathscr{E}}$-maximal rewriting of $E_{0}$ wrt $\mathscr{E}$. By Theorem 2.1 the constructed rewriting is also $\Sigma$-maximal.

The method is based on the idea of characterizing by means of an automaton, which we call $A^{\prime}$, exactly those $\Sigma_{\mathscr{E}}$-words that are not in any rewriting of $E_{0}$ wrt $\mathscr{E}$. Observe that a $\Sigma_{\mathscr{E}}$-word $e_{1} \cdots e_{n}$ is not in any rewriting of $E_{0}$ wrt $\mathscr{E}$ if there is a $\Sigma$-word in its expansion that is not in $L\left(E_{0}\right)$. If we can build such an automaton $A^{\prime}$, then its complement is the maximal rewriting we are looking for, in the sense that it accepts exactly those $\Sigma_{\mathscr{E}}$-words whose expansions are contained in $L\left(E_{0}\right)$. 


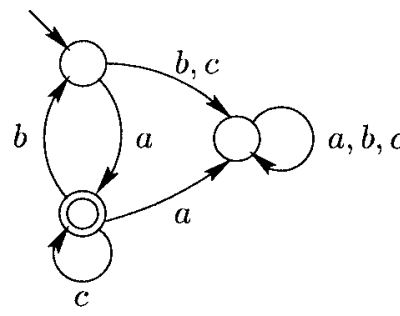

$A_{d}$

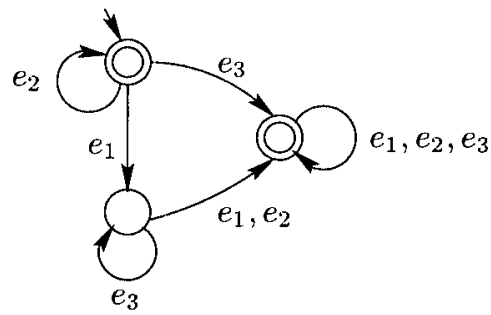

$A^{\prime}$

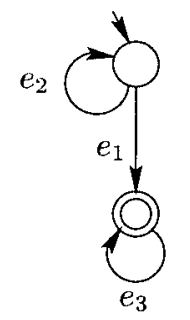

$\overline{A^{\prime}}$

FIG. 1. Construction of the rewriting of $a \cdot(b \cdot a+c)^{*}$ wrt $\left\{a, a \cdot c^{*} \cdot b, c\right\}$.

The crucial point is the construction of $A^{\prime}$. Intuitively, we start from an automaton $A_{d}$ for $E_{0}$ and let $A^{\prime}$ have the same states as $A_{d}$. With regard to the transitions of $A^{\prime}$, we place in $A^{\prime}$ a $\Sigma_{\mathscr{E}}$-edge $e$ between two states $s_{i}$ and $s_{j}$ if there is a $\Sigma$-word in the expansion of $e$ that leads from $s_{i}$ to $s_{j}$ in $A_{d}$. Now, in $A^{\prime}$ a $\Sigma_{\mathscr{E}}$-word $e_{1} \cdots e_{n}$ leads from $s$ to $s^{\prime}$ if in $A_{d}$ there is a sequence of $\Sigma$-words $w_{1} \cdots w_{n}$ that leads from $s$ to $s^{\prime}$. Hence we should let $A^{\prime}$ accept only those $\Sigma_{\mathscr{E}}$-words that lead from the initial state to a state that is non-final for $A_{d}$. However, to guarantee that each $\Sigma$-word in the expansion of $e_{1} \cdots e_{n}$ does not lead also to a final state of $A_{d}$ (and hence is in $\left.L\left(E_{0}\right)\right)$, the automaton $A_{d}$ we start from must be deterministic.

Based on this idea, the construction takes $E_{0}$ and $\mathscr{E}$ as input, and returns an automaton $R_{\mathscr{E}, E_{0}}$ built as follows:

1. Construct a deterministic automaton $A_{d}=\left(\Sigma, S, s_{0}, \rho, F\right)$ such that $L\left(A_{d}\right)=L\left(E_{0}\right)$.

2. Define the automaton $A^{\prime}=\left(\Sigma_{\mathscr{E}}, S, s_{0}, \rho^{\prime}, S-F\right)$, where $s_{j} \in \rho^{\prime}\left(s_{i}, e\right)$ if and only if there exists a word $w \in L(r e(e))$ such that $s_{j} \in \rho^{*}\left(s_{i}, w\right){ }^{1}$. In other words, $A^{\prime}$ has the same states as $A_{d}$, the same initial state $s_{0}$, and as final states all states that are not final in $A_{d}$. With regard to the transitions, $A^{\prime}$ has a transition from $s_{i}$ to $s_{j}$ labeled with $e \in \Sigma_{\mathscr{E}}$ if and only if there is a $\Sigma$-word in the expansion of $e$ that leads from $s_{i}$ to $s_{j}$ in $A_{d}$.

3. $R_{\mathscr{E}, E_{0}}=\overline{A^{\prime}}$, i.e., $R_{\mathscr{E}, E_{0}}$ is the complement of $A^{\prime}$.

Step 2 of the construction requires to check whether there exists a word $w \in L(r e(e))$ such that $s_{j} \in \rho^{*}\left(s_{i}, w\right)$. To do so, we consider the automaton $A_{d}^{i, j}=\left(\Sigma, S, s_{i}, \rho,\left\{s_{j}\right\}\right)$, obtained from $A_{d}$ by suitably changing the initial and final states, and check the product automaton between $A_{d}^{i, j}$ and an automaton for $L(r e(e))$ for non-emptiness.

We illustrate the construction by means of an example.

EXAMPLE 2.2. Let $E_{0}=a \cdot(b \cdot a+c)^{*}, \mathscr{E}=\left\{a, a \cdot c^{*} \cdot b, c\right\}$, and $\Sigma_{\mathscr{E}}=\left\{e_{1}, e_{2}, e_{3}\right\}$, with $r e\left(e_{1}\right)=a, r e\left(e_{2}\right)=a \cdot c^{*} \cdot b$, and $r e\left(e_{3}\right)=c$. The deterministic automaton $A_{d}$ shown in Fig. 1 accepts $L\left(E_{0}\right)$, while $A^{\prime}$ is the corresponding automaton constructed in Step 2 of the rewriting algorithm. Since $A^{\prime}$ is deterministic, by simply exchanging

${ }^{1} \rho^{*}$ denotes the extension of the transition function $\rho$ to words, defined in the standard way for finite automata [HU79]. 
final and nonfinal states we obtain its complement $\overline{A^{\prime}}$, which is the automaton $R_{\mathscr{E}, E_{0}}$ computed by the algorithm.

The following theorem states the correctness of the above construction.

THEOREM 2.2. The automaton $R_{\mathscr{E}, E_{0}}$ is a $\Sigma_{\mathscr{E}}$-maximal rewriting of $E_{0}$ wrt $\mathscr{E}$.

Proof. We first show that $R_{\mathscr{E}, E_{0}}=\overline{A^{\prime}}$ is a rewriting of $E_{0}$ wrt $\mathscr{E}$. If $A^{\prime}$ accepts a $\Sigma_{\mathscr{E}}$-word $e_{1} \cdots e_{n}$, then there exist $n \Sigma$-words $w_{1}, \ldots, w_{n}$ such that $w_{i} \in L\left(r e\left(e_{i}\right)\right)$ for $i=1, \ldots, n$ and such that the $\Sigma$-word $w_{1} \cdots w_{n}$ leads to a non-final state of $A_{d}$. Since $A_{d}$ is deterministic, the fact that $w_{1} \cdots w_{n}$ leads to a non-final state means that it is rejected by $A_{d}$. On the other hand if there exist $n \Sigma$-words $w_{1}, \ldots, w_{n}$ such that $w_{i} \in L\left(r e\left(e_{i}\right)\right)$, for $i=1, \ldots, n$, and $w_{1} \cdots w_{n}$ is rejected by $A_{d}$, then the $\Sigma_{\mathscr{E}}$-word $e_{1} \cdots e_{n}$ is accepted by $A^{\prime}$. That is, $A^{\prime}$ accepts a $\Sigma_{\mathscr{E}}$-word $e_{1} \cdots e_{n}$ if and only if there is a $\Sigma$-word in $\exp _{\Sigma}\left(\left\{e_{1} \cdots e_{n}\right\}\right)$ that is rejected by $A_{d}$. Hence, $R_{\mathscr{E}, E_{0}}$, being the complement of $A^{\prime}$, accepts a $\Sigma_{\mathscr{E}}$-word $e_{1} \cdots e_{n}$ if and only if all $\Sigma$-words $w_{1} \cdots w_{n}$ such that $w_{i} \in L\left(\operatorname{re}\left(e_{i}\right)\right)$ for $i=1, \ldots, n$, are accepted by $A_{d}$. It follows that $R_{\mathscr{E}, E_{0}}$ is a rewriting of $E_{0}$ wrt $\mathscr{E}$.

Next we prove by contradiction that $R_{\mathscr{E}, E_{0}}$ is $\Sigma_{\mathscr{E}}$-maximal. Let $R$ be a rewriting of $E_{0} \quad$ wrt $\mathscr{E}$ such that $L(R) \nsubseteq L\left(\overline{A^{\prime}}\right)$. Let $e_{1} \cdots e_{n}$ be a $\Sigma_{\mathscr{E}}$-word such that $e_{1} \cdots e_{n} \in L(R)$ but $e_{1} \cdots e_{n} \notin L\left(\overline{A^{\prime}}\right)$. By definition of rewriting, all $\Sigma$-words $w_{1} \cdots w_{n}$ such that $w_{i} \in L\left(r e\left(e_{i}\right)\right)$ for $i=1, \ldots, n$, are in $L\left(E_{0}\right)=L\left(A_{d}\right)$. On the other hand, since $e_{1} \cdots e_{n} \notin L\left(\overline{A^{\prime}}\right)$, the $\Sigma_{\mathscr{E}}$-word $e_{1} \cdots e_{n}$ is accepted by $A^{\prime}$. Thus there is a $\Sigma$-word $w_{1} \cdots w_{n}$, such that $w_{i} \in L\left(r e\left(e_{i}\right)\right)$ for $i=1, \ldots, n$, that is rejected by $A_{d}$. Contradiction.

Notably, although Definition 2.1 does not constrain in any way the form of the rewritings, Theorem 2.2 shows that the language over $\Sigma_{\mathscr{E}}$ (and therefore also the language over $\Sigma$ ) defined by the $\Sigma_{\mathscr{E}}$-maximal rewritings is in fact regular (indeed, $\overline{A^{\prime}}$ is a finite automaton).

Next we address the problem of verifying whether the rewriting $R_{\mathscr{E}, E_{0}}$ captures exactly the language defined by $E_{0}$.

Definition 2.3. A rewriting $R$ of $E_{0}$ wrt $\mathscr{E}$ is exact if $\exp _{\Sigma}(L(R))=L\left(E_{0}\right)$.

To verify whether $R_{\mathscr{E}, E_{0}}$ is an exact rewriting of $E_{0}$ wrt $\mathscr{E}$ we proceed as follows:

1. We construct an automaton $B$ over $\Sigma$ that accepts $\exp _{\Sigma}\left(L\left(R_{\mathscr{E}, E_{0}}\right)\right)$ as follows.

We first construct an automaton $A_{i}$ such that $L\left(A_{i}\right)=L\left(\operatorname{re}\left(e_{i}\right)\right)$ for $i=1, \ldots, k$. We assume, without loss of generality, that $A_{i}$ has unique start state and accepting state, and that the start state has no incoming edges and the accepting state no outgoing edges.

We then obtain $B$ by replacing each edge labeled by $e_{i}$ in $R_{\mathscr{E}, E_{0}}$ by a fresh copy of $A_{i}$, identifying the start state of the fresh copy with the source of the edge, and the accepting state with the target of the edge.

Observe that, since $R_{\mathscr{\delta}, E_{0}}$ is a rewriting of $E_{0}, L(B) \subseteq L\left(A_{d}\right)$.

2. We check whether $L\left(A_{d}\right) \subseteq L(B)$, that is, we check whether $L\left(A_{d} \cap \bar{B}\right)=\varnothing$. 
THEOREM 2.3. The automaton $R_{\mathscr{E}, E_{0}}$ is an exact rewriting of $E_{0}$ wrt $\mathscr{E}$ if and only if $L\left(A_{d} \cap \bar{B}\right)=\varnothing$.

Proof. By Theorem 2.2 the automaton $R_{\mathscr{E}, E_{0}}$ is a rewriting of $E_{0}$ wrt $\mathscr{E}$. Suppose $L\left(A_{d} \cap \bar{B}\right)=\varnothing$. Then any $\Sigma$-word $w \in L\left(E_{0}\right)=L\left(A_{d}\right)$ is also accepted by $B$. Hence by construction of $B$ there is a $\Sigma_{\mathscr{E}}$-word $e_{1} \cdots e_{n} \in L\left(\overline{A^{\prime}}\right)$ such that $w=w_{1} \cdots w_{n}$ and $w_{i} \in L\left(r e\left(e_{i}\right)\right)$ for $i=1, \ldots, n$. Suppose that $L\left(A_{d} \cap \bar{B}\right) \neq \varnothing$. Then there exists a $\Sigma$-word $w \in L\left(E_{0}\right)=L\left(A_{d}\right)$ that is rejected by $B$. Hence by construction of $B$ there is no $\sum_{\mathscr{E}}$-word $e_{1} \cdots e_{n} \in L\left(\overline{A^{\prime}}\right)$ such that $w=w_{1} \cdots w_{n}$ and $w_{i} \in L\left(\operatorname{re}\left(e_{i}\right)\right)$ for $i=1, \ldots, n$.

COROLlary 2.1. An exact rewriting of $E_{0}$ wrt $\mathscr{E}$ exists if and only if $L\left(A_{d} \cap \bar{B}\right)=\varnothing$.

EXAMPLE 2.3. Referring to Example 2.2, one can easily verify that $R_{\mathscr{E}, E_{0}}=e_{2}^{*} \cdot e_{1} \cdot e_{3}^{*}$ is exact. Observe that, if $\mathscr{E}$ did not include $c$, the rewriting algorithm would give us $e_{2}^{*} \cdot e_{1}$ as the $\Sigma_{\mathscr{E}}$-maximal rewriting of $E_{0}$ wrt $\left\{a, a \cdot c^{*} \cdot b\right\}$, which, however, is not exact.

\section{COMPLEXITY ANALYSIS}

In this section we analyze the computational complexity of both the problem of rewriting regular expressions, and the method described in Section 2.

\subsection{Upper Bounds}

Let us analyze the complexity of the algorithms presented above for computing the maximal rewriting of a regular expression. By considering the cost of the various steps in computing $R_{\mathscr{E}, E_{0}}$, we immediately derive the following theorem.

THEOREM 3.1. The problem of generating the $\Sigma_{\mathscr{E}}$-maximal rewriting of a regular expression $E_{0}$ wrt a set $\mathscr{E}$ of regular expressions is in 2 EXPTIME.

Proof. We refer to the algorithm that computes $R_{\mathscr{E}, E_{0}}$, and we observe that: (i) Generating the deterministic automaton $A_{d}$ from $E_{0}$ is exponential. (ii) Building $A^{\prime}$ from $A_{d}$ and the expressions $E_{1}, \ldots, E_{k}$ is polynomial. (iii) Complementing $A^{\prime}$ is again exponential.

With regard to the cost of verifying the existence of an exact rewriting, Corollary 2.1 ensures us that we can solve the problem by checking $L\left(A_{d} \cap \bar{B}\right)=\varnothing$. Observe that, if we construct $L\left(A_{d} \cap \bar{B}\right)$, we get a cost of 3EXPTIME, since $\bar{B}$ is of triply exponential size with respect to the size of the input. However, we can avoid the explicit construction of $\bar{B}$, thus getting the following result.

THEOREM 3.2. The problem of verifying the existence of an exact rewriting of a regular expression $E_{0}$ wrt a set $\mathscr{E}$ of regular expressions is in 2 EXPSPACE.

Proof. We refer to the algorithm that verifies whether the automaton $R_{\mathscr{E}, E_{0}}$ is an exact rewriting of $E_{0}$ wrt $\mathscr{E}$, and we observe that: (i) By Theorem 3.1, the automaton $R_{\mathscr{E}, E_{0}}$ is of doubly exponential size. (ii) Building the automaton $B$ from $R_{\mathscr{E}, E_{0}}$ is 
polynomial. (iii) Complementing $B$ to get $\bar{B}$ is exponential. (iv) Verifying the emptiness of the intersection of $A_{d}$ and $\bar{B}$ can be done in nondeterministic logarithmic space [RS59, Jon75]. Combining (i)-(iv), we get a nondeterministic 2EXPSPACE bound, and using Savitch's Theorem [Sav70], we get a deterministic 2EXPSPACE bound.

Some care, however, is needed to getting the claimed space bound. We cannot simply construct $\bar{B}$, since it is of triply exponential size. Instead, we construct $\bar{B}$ "onthe-fly"; whenever the nonemptiness algorithm wants to move from a state $s_{1}$ of the intersection of $A_{d}$ and $\bar{B}$ to a state $s_{2}$, the algorithm guesses $s_{2}$ and checks that it is directly connected to $s_{1}$. Once this has been verified, the algorithm can discard $s_{1}$. Thus, at each step the algorithm needs to keep in memory at most two states and there is no need to generate all of $\bar{B}$ at any single step of the algorithm.

\subsection{Lower Bounds}

We show that the upper bounds established in Section 3.1 are essentially optimal. To prove the matching lower bounds we exploit variants of tiling problems (see e.g., [vEB82, vEB97, Ber66]). A tile is a unit square of one of several types and a tiling system is specified by means of a finite set $\Delta$ of tile types and two binary relations $H$ and $V$ over $\Delta$, representing horizontal and vertical adjacency relations, respectively. A generic tiling problem consists in determining whether there exists a mapping $\tau$ (called tiling) from a given region $R$ of the integer plane to $\Delta$ which is consistent with $H$ and $V$. That is, if $(i, j),(i, j+1) \in R$ then $(\tau(i, j), \tau(i, j+1)) \in H$ and if $(i, j),(i+1, j) \in R$ then $(\tau(i, j), \tau(i+1, j)) \in V$. We get a specific tiling problem by imposing additional conditions on the region to be tiled and on the tile types that can be placed in certain positions of the region, such as the first/last row/column, or the borders.

Different tiling problems have been shown to be complete for various complexity classes [vEB82, vEB97]. We will use EXPSPACE and 2EXPSPACE-complete tiling problems.

\subsubsection{Existence of a nonempty rewriting}

We say that a rewriting $R$ is $\Sigma_{\mathscr{E}}$-empty if $L(R)=\varnothing$. We say that it is $\Sigma$-empty if $\exp _{\Sigma}(L(R))=\varnothing$. Clearly $\Sigma_{\mathscr{E}}$-emptiness implies $\Sigma$-emptiness. The converse also holds except for the non-interesting case where $\mathscr{E}$ contains one or more expressions $E$ such that $L(E)=\varnothing$. Therefore, we will talk about the emptiness of a rewriting $R$ without distinguishing between the two definitions.

We consider the tiling problem $T=\left(\Delta, H, V, t_{S}, t_{F}, C_{E S}\right)$, where $t_{S}$ and $t_{F}$ are two distinguished tile types in $\Delta$, and for a given number $n$ in unary, $C_{E S}$ requires to tile a region of size $2^{n} \times k$, for some number $k$, in such a way that the left bottom tile of the region (i.e., the one in position $(0,0)$ ) is of type $t_{S}$ and the right upper tile (i.e., the one in position $\left.\left(2^{n}-1, k-1\right)\right)$ is of type $t_{F}$. Using a reduction from acceptance of EXPSPACE Turing machines analogous to the one in [vEB97], it can be shown that this variant of tiling problem is EXPSPACE-complete. 
We exploit such a tiling problem to prove the EXPSPACE lower bound of the problem of verifying the existence of a nonempty rewriting. That is, given an instance $T$ of the above tiling problem and a number $n$, we construct a regular expression $E_{0}$ and a set $\mathscr{E}$ of regular expressions such that a tiling corresponding to $T$ (a $T$-tiling) exists if and only if there is a nonempty rewriting of $E_{0}$ wrt $\mathscr{E}$.

A tiling of a region of size $2^{n} \times k$ can be described as a word over $\Delta$ of length $k 2^{n}$, where every block of $2^{n}$ symbols describes a row of the tiling. We take $\Sigma_{\mathscr{E}}$ to be $\Delta$. We will define $E_{0}$ and $r e(e)$ for each letter $e \in \Delta$ such that a $\Delta$-word $e_{1} \cdots e_{\ell}$ describes a $T$-tiling if and only if $\exp _{\Sigma}\left(e_{1} \cdots e_{\ell}\right) \subseteq L\left(E_{0}\right) . E_{0}$ will be defined as the sum $E_{\text {bad }}+E_{\text {good }}$ of two regular expressions $E_{\text {bad }}$ and $E_{\text {good }}$, which are in turn defined as sums of regular expressions.

The construction of $r e(e)$ for $e \in \Delta$ is uniform: we take the alphabet $\Sigma$ to be $\Delta \cup\{0,1, \$\}$ (so $\Sigma_{\mathscr{E}} \subseteq \Sigma$ ), and define $r e(e)=\$ \cdot(0+1)^{3 n+1} \cdot e$; that is, the language associated with $e$ consists of $e$ prefixed with a $\$$ sign and all binary words of length $3 n+1$. Intuitively, the $\$$ sign is a marker, the first $n$ bits encode the column of a tile ( $n$ bits are needed to describe the column in a row of length $2^{n}$ ), and the next $2 n$ bits encode bookkeeping information. The $3 n+1$-st bit is a highlight. As will become clear shortly, highlights are used to identify either a tile not in the last column or a pair of vertically adjacent tiles. Given a word $w \in L(r e(e))$, we use

- $\operatorname{position}(w)$ to denote the first $n$ bits after the $\$$ marker,

- $\operatorname{carry}(w)$ to denote the second $n$ bits after the $\$$ marker, and

- $n \operatorname{ext}(w)$ to denote the third $n$ bits after the $\$$ marker.

Also, we use position $(w, i), \operatorname{carry}(w, i)$ and $n \operatorname{ext}(w, i)$, for $0 \leqslant i<n$ to denote the $i+1$-st bit in position $(w), \operatorname{carr} y(w)$, and $n \operatorname{ext}(w)$, respectively. This means that we count bits starting from 0 and consider the least significant bit to be the one in position 0 .

Consider now a word $e_{0} \cdots e_{\ell}$ over $\Delta$, and let $w=w_{0} \cdots w_{\ell}$ be a word in $\exp _{\Sigma}\left(e_{0} \cdots e_{\ell}\right)$. We call each $w_{j}$, which is a word of length $3 n+3$, a block. We classify such words $w$ into two classes. Our intention is that position $\left(w_{j}\right)$ describes an $n$-bit counter, and that precisely one or two highlight bits are on. When only one highlight bit is on it is located in a block $w_{h}$ such that position $\left(w_{h}\right) \neq 1^{n}$, and when two highlight bits are on, they are located in blocks $w_{h}$ and $w_{k}$ such that position $\left(w_{h}\right)=\operatorname{position}\left(w_{k}\right)$ and for at most one $j, h<j<k$, we have position $\left(w_{j}\right)=0^{n}$. Requiring position $\left(w_{j}\right)$ to be an $n$-bit counter means that we expect position $\left(w_{0}\right)=0^{n}$ and position $\left(w_{\ell}\right)=1^{n}$, and we expect $\operatorname{carry}\left(w_{j}\right)$ to be the sequence of $n$ carry bits when position $\left(w_{j}\right)$ is incremented to yield next $\left(w_{j}\right)$, which is equal to position $\left(w_{j+1}\right)$. If the intended conditions do not hold, then $w$ is a bad word. More precisely, a word $w=w_{0} \cdots w_{\ell}$ is bad if one of the following holds:

1. $\operatorname{position}\left(w_{0}, i\right)=1$, for some $i, 0 \leqslant i<n$;

2. $\operatorname{position}\left(w_{\ell}, i\right)=0$, for some $i, 0 \leqslant i<n$;

3. $\operatorname{carry}\left(w_{j}, 0\right)=0$, for some $j, 0 \leqslant j \leqslant \ell$;

4. $\operatorname{carry}\left(w_{j}, i\right) \neq \operatorname{carry}\left(w_{j}, i-1\right)$ and $\operatorname{position}\left(w_{j}, i-1\right)$, for some $j$ and $i$, $0 \leqslant j \leqslant \ell, 1 \leqslant i<n$; 
5. $n \operatorname{ext}\left(w_{j}, i\right) \neq \operatorname{position}\left(w_{j}, i\right)$ xor $\operatorname{carry}\left(w_{j}, i\right)$, for some $j$ and $i, 0 \leqslant j \leqslant \ell$, $0 \leqslant i<n$;

6. $\operatorname{position}\left(w_{j}, i\right) \neq n \operatorname{ext}\left(w_{j-1}, i\right)$, for some $j$ and $i, 1 \leqslant j \leqslant \ell, 0 \leqslant i<n$;

7. conditions on the highlight bits, which are:

(i) no highlight bit in $w$ is 1 ;

(ii) only one highlight bit in $w$ is 1 and it is located in a block $w_{h}$ such that $\operatorname{position}\left(w_{h}\right)=1^{n}$;

(iii) at least three highlight bits in $w$ are 1;

(iv) the two highlight bits that are 1 are located in two blocks $w_{h}$ and $w_{k}$ and there are at least two blocks $w_{j_{1}}$ and $w_{j_{2}}$ between $w_{h}$ and $w_{k}$ such that $\operatorname{position}\left(w_{j_{1}}\right)=\operatorname{position}\left(w_{j_{2}}\right)=0^{n}$;

(v) the two highlight bits that are 1 are located in two blocks $w_{h}$ and $w_{k}$ and $\operatorname{position}\left(w_{h}, i\right) \neq \operatorname{position}\left(w_{k}, i\right)$ for some $i, 0 \leqslant i<n$.

We define $E_{\text {bad }}$ in such a way that all bad words belong to $L\left(E_{\mathrm{bad}}\right)$. Each of the above conditions can be "detected" by a regular expression of size polynomial in $n$, which contributes to $E_{\mathrm{bad}}$ (and hence to $E_{0}$ ). To illustrate the idea, we provide the regular expressions for some of the conditions above.

Condition (1) is detected by the regular expression

$$
\left(\sum_{i=0}^{n-1} \$ \cdot(0+1)^{i} \cdot 1 \cdot(0+1)^{3 n-i} \cdot \Delta\right) \cdot B^{*}
$$

where $B$ stands for the regular expression $\$ \cdot(0+1)^{3 n+1} \cdot \Delta$.

Condition (4) is detected by the sum of four regular expressions

$$
\begin{aligned}
B^{*} \cdot\left(\sum_{i=1}^{n-1} \$ \cdot(0+1)^{i-1} \cdot p \cdot(0+1)^{n-i}\right. \\
\left.\cdot(0+1)^{i-1} \cdot c \cdot c^{\prime}(0+1)^{n-1-i} \cdot(0+1)^{n+1} \cdot \Delta\right) \cdot B^{*}
\end{aligned}
$$

one for each choice of 0 or 1 for $p, c$, and $c^{\prime}$ such that $c^{\prime} \neq c$ and $p$.

Condition (6) is detected by the sum of two regular expressions

$$
\begin{aligned}
B^{*} \cdot\left(\sum_{i=0}^{n-1} \$ \cdot(0+1)^{2 n} \cdot(0+1)^{i} \cdot b \cdot(0+1)^{n-1-i} \cdot(0+1) \cdot \Delta\right. \\
\left.\cdot \$ \cdot(0+1)^{i} \cdot \bar{b} \cdot(0+1)^{n-1-i} \cdot(0+1)^{2 n} \cdot(0+1) \cdot \Delta\right) \cdot B^{*}
\end{aligned}
$$

one for $b=0$ and $\bar{b}=1$, and one for $b=1$ and $\bar{b}=0$.

Condition (7-ii) is detected by the regular expression

$$
\left(\$ \cdot(0+1)^{3 n} \cdot 0 \cdot \Delta\right)^{*} \cdot \$ \cdot 1^{n} \cdot(0+1)^{2 n} \cdot 1 \cdot \Delta \cdot\left(\$ \cdot(0+1)^{3 n} \cdot 0 \cdot \Delta\right)^{*}
$$


Condition (7-v) is detected by the sum of two regular expressions

$$
\begin{aligned}
B^{*} \cdot\left(\sum_{i=0}^{n-1} \$ \cdot(0+1)^{i} \cdot b \cdot(0+1)^{3 n-1-i} \cdot 1 \cdot \Delta \cdot B^{*}\right. \\
\left.\cdot \$ \cdot(0+1)^{i} \cdot \bar{b} \cdot(0+1)^{3 n-1-i} \cdot 1 \cdot \Delta\right) \cdot B^{*}
\end{aligned}
$$

one for $b=0$ and $\bar{b}=1$, and one for $b=1$ and $\bar{b}=0$.

Words that satisfy none of the above conditions are good words, and will be handled differently. In such words either one or two highlight bits are on. When one highlight bit is on, it is located at a block that corresponds to a tile not in the last column in a tiling of the region. The types of this tile and of the one immediately to the right have to be related in a way that depends on the horizontal adjacency relation $H$ of $T$. When two highlight bits are on, they are located at two positions that are precisely $2^{n}$ blocks apart, and these blocks correspond to vertically adjacent tiles. The types of these tiles have to be related in a way that depends on the vertical adjacency relation $V$ of $T$. We can use regular expressions of size polynomial in $n$ to force such blocks to be related in the right way, and also to force the tiling to satisfy the additional conditions on the left bottom and right upper tiles. $E_{\text {good }}$ is the sum of all such regular expressions.

For example, the following regular expression ensures that the horizontal adjacency relation is respected in the case where the highlight bit is on at a block that is neither the first nor the last one:

$$
\begin{aligned}
\$ \cdot(0+1)^{3 n} \cdot 0 \cdot t_{S} \cdot\left(\$ \cdot(0+1)^{3 n} \cdot 0 \cdot \Delta\right)^{*} \\
\quad\left(\sum_{\left(t_{1}, t_{2}\right) \in H} \$ \cdot(0+1)^{3 n} \cdot 1 \cdot t_{1} \cdot \$ \cdot(0+1)^{3 n} \cdot 0 \cdot t_{2}\right) \\
\cdot\left(\$ \cdot(0+1)^{3 n} \cdot 0 \cdot \Delta\right)^{*} \cdot \$ \cdot(0+1)^{3 n} \cdot 0 \cdot t_{F} \cdot
\end{aligned}
$$

The following regular expression ensures that the vertical adjacency relation is respected in the case where the two highlight bits are on at blocks that are neither the first nor the last one:

$$
\begin{aligned}
\$ \cdot(0+1)^{3 n} \cdot 0 \cdot t_{S} \cdot\left(\$ \cdot(0+1)^{3 n} \cdot 0 \cdot \Delta\right)^{*} \\
\quad\left(\sum_{\left(t_{1}, t_{2}\right) \in V} \$ \cdot(0+1)^{3 n} \cdot 1 \cdot t_{1} \cdot\left(\$ \cdot(0+1)^{3 n} \cdot 0 \cdot \Delta\right)^{*} \cdot \$ \cdot(0+1)^{3 n} \cdot 1 \cdot t_{2}\right) \\
\quad \cdot\left(\$ \cdot(0+1)^{3 n} \cdot 0 \cdot \Delta\right)^{*} \cdot \$ \cdot(0+1)^{3 n} \cdot 0 \cdot t_{F} .
\end{aligned}
$$

Similar regular expressions can be provided for the cases where the highlight bits are on at the first or last block.

Thus, all the good words $w=w_{0} \cdots w_{\ell}$ in $\exp _{\Sigma}\left(e_{0} \cdots e_{\ell}\right)$ are in $L\left(E_{\text {good }}\right)$ if and only if $e_{0} \cdots e_{\ell}$ describes a $T$-tiling. If no $T$-tiling exists then for every $e_{0} \cdots e_{\ell}$ we can find a good word $w=w_{0} \cdots w_{\ell}$ in $\exp _{\Sigma}\left(e_{0} \cdots e_{\ell}\right)$ that is not in $L\left(E_{\text {good }}\right)$ and hence not in $L\left(E_{0}\right)$. Thus, $E_{0}$ has a nonempty rewriting wrt $\mathscr{E}$ if and only if a $T$-tiling exists. 
THEOREM 3.3. The problem of verifying the existence of a nonempty rewriting of a regular expression $E_{0}$ wrt a set $\mathscr{E}$ of regular expressions is EXPSPACE-complete.

Proof. By Theorem 3.1, we generate the $\Sigma_{\mathscr{E}}$-maximal rewriting of a regular expression $E_{0}$ wrt a set $\mathscr{E}$ of regular expressions in 2EXPTIME. Checking whether a given finite-state automaton is non-empty can be done in NLOGSPACE. The upper bound follows (see comments in the proof of Theorem 3.2). The lower bound follows from the reduction from the EXPSPACE-complete tiling problem described above, by observing that $E_{0}$ and all regular expressions in $\mathscr{E}$ are of size polynomial in $T$ and $n$.

Note that Theorem 3.3 implies that the upper bound established in Theorem 3.1 is essentially optimal. If we can generate maximal rewritings in, say, EXPTIME, then we could test emptiness in PSPACE, which is impossible by Theorem 3.3. We can get, however, an even sharper lower bound on the size of rewritings.

THEOREM 3.4. For each $n>0$ there is a regular expression $E_{0}^{n}$ and a set $\mathscr{E}^{n}$ of regular expressions such that the combined size of $E_{0}^{n}$ and $\mathscr{E}^{n}$ is polynomial in $n$, but the shortest nonempty rewriting (expressed either as a regular expression or as an automaton) of $E_{0}^{n}$ wrt $\mathscr{E}^{n}$ is of length $2^{2^{n}}$.

Proof. We use the encoding technique of Theorem 3.3. Instead, however, of encoding tiling problems, we directly encode a $2^{n}$-bit counter using an alphabet $\Sigma_{\mathscr{E}}=\left\{b_{000}, b_{001}, \ldots, b_{111}\right\}$ of 8 symbols representing the 8 possible combinations of a position, a carry, and a next bit. For a symbol $b_{p c x}$, where $p, c, x \in\{0,1\}$, we say that $p$ is the position-component, $c$ the carry-component, and $x$ the next-component of $b_{p c x}$. In a word over $\Sigma_{\mathscr{E}}$ representing the evolution of the $2^{n}$-bit counter, the three components of symbols that are exactly $2^{n}$ positions apart will represent the position, carry, and next bits in the same position of two successive configurations of the counter. By using the highlight bits of the encoding technique of Theorem 3.3 we can enforce the correct relationships between such symbols. Hence, we can define $E_{0}^{n}$ and the set $\mathscr{E}^{n}=\left\{B_{000}^{n}, \ldots, B_{111}^{n}\right\}$ of regular expressions associated to the symbols in $\Sigma_{\mathscr{E}}$ in such a way that a word $w=b_{p_{0} c_{0} x_{0}} \cdots b_{p_{m} c_{m} x_{m}}$ is a rewriting of $E_{0}^{n}$ wrt $\mathscr{E}^{n}$ if and only if the bit vector $p_{0} \cdots p_{m}$ represented by the position-components of $w$ is of the form $w_{0} \cdots w_{2^{2^{n}}-1}$, where $w_{j}$ is the $2^{n}$-bit representation of $j$.

Using pumping arguments it is easy to see that any regular expression or automaton describing such a rewriting has to be of length at least $2^{2^{n}}$. Indeed, assume there is a regular expression or automaton $R$ of size less than $2^{2^{n}}$ describing the rewriting. Then, since any nonempty regular expression or automaton accepts at least one word of length less than or equal to its size, $R$ accepts also a word $w^{\prime}$ of length less than $2^{2^{n}}$, contradicting the hypothesis that $R$ is a correct rewriting of $E_{0}^{n}$ wrt $\mathscr{E}^{n}$.

\subsubsection{Existence of an Exact Rewriting}

The technique used in Theorem 3.3 turns out to be an important building block in the proof that Theorem 3.2 is also tight. 
We consider the tiling problem $T=\left(\Delta, H, V, t_{S}, t_{F}, t_{L}, t_{R}, C_{2 E S}\right)$, where $t_{S}, t_{F}, t_{L}$, and $t_{R}$ are distinguished tile types in $\Delta$ such that $\left(t_{R}, t_{L}\right) \in H$, and for a given number $n$ in unary, $C_{2 E S}$ requires to tile a region of size $2^{2^{n}} \times k$, for some number $k$, in such a way that: (i) the left bottom tile of the region is of type $t_{S}$, (ii) all other tiles on the left border are of type $t_{L}$, (iii) the right upper tile is of type $t_{F}$, and (iv) all other tiles on the right border are of type $t_{R}$. Intuitively, $t_{S}$ corresponds to the initial state of a Turing machine on the first tape position, $t_{F}$ corresponds to the final state on the last tape position reached by the machine in the final configuration, and $t_{L}$ and $t_{R}$ correspond respectively to left and right endmarkers of Turing machine configurations. Using a reduction from acceptance of 2EXPSPACE Turing machines analogous to the one in [vEB97], it can be shown that this tiling problem is 2EXPSPACE-complete.

We exploit such a tiling problem to prove the 2EXPSPACE lower bound of the problem of verifying the existence of an exact rewriting. That is, given an instance $T$ of the above tiling problem and a number $n$, we construct a regular expression $E_{0}$ and a set $\mathscr{E}$ of regular expressions such that a $T$-tiling exists if and only if there is an exact rewriting of $E_{0}$ wrt $\mathscr{E}$. Each row of a $T$-tiling is of doubly exponential length in $n$. We describe such a tiling as a word over $\Delta$, and to "check" the vertical adjacency conditions we need to compare the types of tiles that are a doubly exponential distance apart, which requires "yardsticks" of such length. Fortunately, we have seen in the proofs of Theorems 3.3 and 3.4 how to construct such yardsticks.

We directly exploit the construction described in Theorem 3.4 to encode a $2^{n}$ bit counter. Let $E_{0}^{C}$ and $\mathscr{E}^{C}$ be respectively the regular expression $E_{0}^{n}$ and the set $\mathscr{E}^{n}=\left\{B_{000}^{n}, \ldots, B_{111}^{n}\right\}$ of regular expressions of Theorem 3.4. Such regular expressions are over the alphabet $\Sigma^{C}=\{0,1, \$\} \cup \Delta^{C}$, where we have denoted with $\Delta^{C}$ the set $\Sigma_{\mathscr{E}}^{C}=\left\{b_{000}, b_{001}, \ldots, b_{111}\right\}$ of 8 symbols representing the 8 possible combinations of a position, a carry, and a next bit of the $2^{n}$ bit counter, used in the proof of Theorem 3.4. Let $r e^{C}(\cdot)$ be the mapping that associates each regular expression in $\mathscr{E}^{C}$ with the corresponding symbol in $\Sigma_{\mathscr{E}}^{C}$. Then for a word $w$ over $\Sigma_{\mathscr{E}}^{C}$ we have that $\exp _{\Sigma}(w) \subseteq L\left(E_{0}^{C}\right)$ precisely when $w=w_{C}$, where $w_{C}$ is the word that describes the $2^{2^{n}}$ successive bit configurations (for the position, the carry and the next bits) of the $2^{n}$ bit counter. In particular, since each bit configuration is of length $2^{n}$, we have that $w_{C}$ is of length $2^{n} \cdot 2^{2^{n}}$, which is precisely what we need. We will use $E_{0}^{C}$ and $\mathscr{E}^{C}$ to construct regular expressions that detect errors in $T$-tilings with rows of length exactly $^{2} 1+2^{n} \cdot 2^{2^{n}}$.

Let $\tilde{\Delta}=\{\tilde{t} \mid t \in \Delta\}$, where $\Delta$ is the set of tile types of $T$. We take $\Sigma$ to be $\Sigma^{C} \cup \tilde{\Delta} \cup \Delta$ and $\Sigma_{\mathscr{E}}$ to be $\Sigma_{\mathscr{E}}^{C} \cup \tilde{\Delta}$. The set $\mathscr{E}$ of regular expressions used for the rewriting is obtained by taking $r e(e)=r e^{C}(e)+\Delta$, for each $e \in \Sigma_{\mathscr{E}}^{C}$, and $r e(\tilde{t})=\tilde{t}+t$, for each $\tilde{t} \in \tilde{\Delta}$. Thus each symbol in $\Sigma_{\mathscr{E}}^{C}$ generates also all possible tile types in $\Delta$, while each symbol in $\tilde{\Delta}$ generates itself and only the corresponding tile type.

We construct regular expressions $E_{0}^{V}, E_{0}^{H}, E_{0}^{S}, E_{0}^{F}, E_{0}^{L}$, and $E_{0}^{R}$, which are used to detect errors in candidate tilings. $E_{0}^{V}$ is used to detect conflicts with respect to the vertical adjacency relation $V$, which arise between tiles that are $1+2^{n} \cdot 2^{2^{n}}$ symbols

${ }^{2}$ We use tilings with rows of length $1+2^{n} \cdot 2^{2^{n}}$ instead of $2^{2^{n}}$ since this simplifies the construction of the regular expressions. 
apart. $E_{0}^{H}$ is used to detect conflicts with respect to the horizontal adjacency relation $H$, which arise between tiles that are directly adjacent. Note that since $\left(t_{R}, t_{L}\right) \in H$, also the last tile of a row and the first tile of the next row have to respect the horizontal adjacency condition. $E_{0}^{S}, E_{0}^{F}, E_{0}^{L}$, and $E_{0}^{R}$ are used to detect tiles of the wrong type at the beginning and end, and on the left and right border respectively. The regular expressions are constructed in such a way that for a word $w$ over $\Sigma_{\mathscr{E}}$ we have that:

- $\exp _{\Sigma}(w) \subseteq L\left(E_{0}^{V}\right)$ precisely when $w$ is in the form

$$
\Sigma_{\delta}^{C *} \cdot\left(\sum_{\left(t_{1}, t_{2}\right) \in \bar{V}} \tilde{t}_{1} \cdot \Sigma_{\delta}^{C} \cdot w_{C} \cdot \tilde{t}_{2}\right) \cdot \Sigma_{\delta}^{C *}
$$

where $\bar{V}$ is the set of pairs of tiles that are not in $V$.

- $\exp _{\Sigma}(w) \subseteq L\left(E_{0}^{H}\right)$ precisely when $w$ is in the form

$$
\Sigma_{\mathscr{E}}^{C *} \cdot\left(\sum_{\left(t_{1}, t_{2}\right) \in \bar{H}} \tilde{t}_{1} \cdot \tilde{t}_{2}\right) \cdot \Sigma_{\mathscr{E}}^{C *}
$$

where $\bar{H}$ is the set of pairs of tiles that are not in $H$.

- $\exp _{\Sigma}(w) \subseteq L\left(E_{0}^{S}\right)$ precisely when $w$ is in the form

$$
\left(\sum_{t \in \Delta \backslash\left\{t_{s}\right\}} \tilde{t}\right) \cdot \Sigma_{\delta}^{C *}
$$

- $\exp _{\Sigma}(w) \subseteq L\left(E_{0}^{F}\right)$ precisely when $w$ is in the form

$$
\left(\Sigma_{\mathscr{E}}^{C} \cdot w_{C}\right)^{*} \cdot w_{C} \cdot\left(\sum_{t \in \Delta \backslash\left\{t_{F}\right\}} \tilde{t}\right)
$$

- $\exp _{\Sigma}(w) \subseteq L\left(E_{0}^{L}\right)$ precisely when $w$ is in the form

$$
\left(\Sigma_{\mathscr{\delta}}^{C} \cdot w_{C}\right)^{*} \cdot \Sigma_{\mathscr{E}}^{C} \cdot w_{C} \cdot\left(\sum_{t \in \Delta \backslash\left\{t_{L}\right\}} \tilde{t}\right) \cdot \Sigma_{\mathscr{\delta}}^{C *}
$$

- $\exp _{\Sigma}(w) \subseteq L\left(E_{0}^{R}\right)$ precisely when $w$ is in the form

$$
\left(\Sigma_{\delta}^{C} \cdot w_{C}\right)^{*} \cdot w_{C} \cdot\left(\sum_{t \in \Delta \backslash\left\{t_{R}\right\}} \tilde{t}\right) \cdot \Sigma_{\delta}^{C} \cdot \Sigma_{\delta}^{C *} .
$$

The construction of $E_{0}^{H}$ and $E_{0}^{S}$ is immediate. For the other regular expressions we need to construct a regular expression $E_{0}^{C A}$, of size polynomial in $n$, whose rewriting is $w_{C}$. We make use of $E_{0}^{C}$ and $\mathscr{E}^{C}$, but need to take into account that, wrt the construction in Theorem 3.4, now a symbol $e$ in $\Sigma_{\&}^{C}$ generates not only all possible sequences of type $\$ \cdot(0+1)^{3 n+1} \cdot e$ (and hence of length $\left.3 n+3\right)$ but also all symbols in $\Delta$. We can however exploit the fact that $E_{0}^{C}$ is composed of subexpressions that 
generate words of length $3 n+3$ and thus obtain $E_{0}^{C \Delta}$ from $E_{0}^{C}$ by simply adding the expression $\Delta$ to each such subexpression. Then we have for example that

$$
E_{0}^{V}=\left(B^{C}+\Delta\right)^{*} \cdot\left(\sum_{\left(t_{1}, t_{2}\right) \in \bar{V}}\left(\tilde{t}_{1}+t_{1}\right) \cdot\left(B^{C}+\Delta\right) \cdot E_{0}^{C \Delta} \cdot\left(\tilde{t}_{2}+t_{2}\right)\right) \cdot\left(B^{C}+\Delta\right)^{*}
$$

where $B^{C}$ stands for the regular expression $\$ \cdot(0+1)^{3 n+1} \cdot \Delta^{C}$. The regular expressions $E_{0}^{F}, E_{0}^{L}$, and $E_{0}^{R}$ are constructed in a similar way.

The regular expression $E_{0}^{1}=E_{0}^{V}+E_{0}^{H}+E_{0}^{S}+E_{0}^{F}+E_{0}^{L}+E_{0}^{R}$ is such that a rewriting of $E_{0}^{1}$ generates only candidate tilings with some error (in addition to words containing also $\$, 0,1$, the symbols in $\Delta^{C}$, and at most two symbols in $\tilde{\Delta}$ ).

To encode the problem of the existence of an exact rewriting, we take $E_{0}$ to be $E_{0}^{1}+\Delta^{*}$, i.e., $E_{0}$ expresses also all "candidate" tilings using the tile types in $\Delta$. If no $T$-tiling exists, then every candidate tiling will have an error, and thus will already be generated by a rewriting of $E_{0}^{1}$. If, on the other hand, a $T$-tiling exists, such a tiling does not have an error and will not be generated by the rewriting of $E_{0}^{1}$, resulting in a non-exact rewriting. Notice that we cannot attempt to construct a rewriting of $\Delta^{*}$ separately, and the only way to get one is via the rewriting of $E_{0}^{1}$. This is due to the fact that, from the symbols in $\Sigma_{\mathscr{E}}=\Sigma_{\mathscr{E}}^{C} \cup \tilde{\Delta}$, each symbol $e$ in $\Sigma_{\mathscr{E}}^{C}$ generates not only all symbols in $\Delta$, but also sequences of type $\$ \cdot(0+1)^{3 n+1} \cdot e$, while each symbol $\tilde{t}$ in $\tilde{\Delta}$ generates besides $t$ also $\tilde{t}$.

THEOREM 3.5. The problem of verifying the existence of an exact rewriting of a regular expression $E_{0}$ wrt a set $\mathscr{E}$ of regular expressions is 2 EXPSPACE-complete.

Proof. The upper bound proof is given in Theorem 3.2. The lower bound follows from the reduction from the 2EXPSPACE complete tiling problem described above, by observing that $E_{0}$ and all regular expressions in $\mathscr{E}$ are of size polynomial in $T$ and $n$.

\section{QUERY REWRITING IN SEMI-STRUCTURED DATA}

In this section we show how to apply the results presented above to query rewriting in semi-structured data.

All semi-structured data models share the characteristic that data are organized in a labeled graph [Bun97, Abi97]. Following this idea two different approaches have been proposed:

1. The first approach associates data both with the nodes and with the edges. Specifically, nodes represent objects, and edges represent relations between objects [Abi97, QRS +95, FFLS97, FFK +98].

2. The second approach associates data with the edges only [BDFS97, BDHS96, FS98], but queries are not expressed directly over the constants labeling the edges of databases, but over logical formulae describing the properties of such edges.

The basic query mechanism in both approaches is that of regular path queries. A regular path query is specified through a regular expression and the answer to such 
a query is a set of pairs of nodes connected in the database through a path conforming to the regular expression.

The rewriting techniques proposed in Section 2 can be applied to rewrite regular path queries in both approaches. In the first approach it can be applied directly. It is sufficient to show that $R$ is a rewriting of a query $Q$ if and only if $R$ (considered as a mechanism to define a language) is a rewriting of the regular expression $Q .{ }^{3}$ In the second approach more care is required due to the fact that queries are over formulae rather than directly over symbols representing data. In the rest of the section we concentrate on this case.

\subsection{Semi-structured Data Models and Queries}

Following [BDFS97], we consider a (semi-structured) database as a graph $D B$ whose edges are labeled by elements from a given domain $\mathscr{D}$, which we assume finite. We denote an edge from node $x$ to node $y$ labeled by $a$ with $x \stackrel{a}{\rightarrow} y$. Typically, a database will be a rooted connected graph; however, in this paper we do not need to make this assumption. In order to define queries over a semi-structured database we start from a decidable, complete ${ }^{4}$ first-order theory $\mathscr{T}$ over the domain $\mathscr{D}$. We assume that the language of $\mathscr{T}$ includes one distinct constant for each element of $\mathscr{D}$ (in the following we do not distinguish between constants and elements of $\mathscr{D}$ ). We further assume that among the predicates of $\mathscr{T}$ we have one unary predicate of the form $\lambda z . z=a$, for each constant $a$ in $\mathscr{D}$. We use simply $a$ as an abbreviation for such predicate. Finally, as in [BDFS97], we consider both the size of $\mathscr{T}$ (and hence the number of constants in $\mathscr{D}$ ), and the time needed to check validity of any formula in $\mathscr{T}$ to be constant.

In this setting, a regular path query (which we call simply query), denotes all the paths corresponding to words of a specified regular language defined over the (finite) set $\mathscr{F}$ of formulae of $\mathscr{T}$ with one free variable. Such formulae are used to describe properties that the labels of the edges of the database must satisfy. Regular path queries are the basic constituents of queries in semi-structured data, and are typically expressed by means of regular expressions [BDHS96, Abi97, FS98, MS99]. Another possibility to express regular path queries is to use finite automata.

When evaluated over a database, a query $Q$ returns the set of pairs of nodes connected by a path that conforms to the regular language $L(Q)$ defined by $Q$, according to the following definitions.

DEFINITION 4.1. Given an $\mathscr{F}$-word $\varphi_{1} \cdots \varphi_{n}$, a $\mathscr{D}$-word $a_{1} \cdots a_{n}$ matches $\varphi_{1} \cdots \varphi_{n}$ (wrt $\mathscr{T}$ ) if and only if $\mathscr{T} \vDash \varphi_{i}\left(a_{i}\right)$, for $i=1, \ldots, n$.

We denote the set of $\mathscr{D}$-words that match an $\mathscr{F}$-word $w$ by $\operatorname{match}(w)$, and, given a language $\ell$ over $\mathscr{F}$, we denote $\bigcup_{w \in \ell} \operatorname{match}(w)$ by $\operatorname{match}(\ell)$.

${ }^{3}$ The proof is similar to that for Theorem 4.1 below.

${ }^{4}$ The theory is complete in the sense that for every closed formula $\varphi$, either $\mathscr{T}$ entails $\varphi$, or $\mathscr{T}$ entails $\neg \varphi$ [BDFS97]. 
Definition 4.2. The answer to a query $Q$ over $a$ database $D B$ is the set ans $(L(Q), D B)$, where for a language $\ell$ over $\mathscr{F}$

$\operatorname{ans}(\ell, D B)=\left\{(x, y) \mid\right.$ there is a path $x \stackrel{a_{1}}{\longrightarrow} \cdots \stackrel{a_{n}}{\longrightarrow} y$ in $D B$ s.t. $\left.a_{1} \cdots a_{n} \in \operatorname{match}(\ell)\right\}$

\subsection{Rewriting Regular Path Queries}

In order to apply the results on rewriting of regular expressions to query rewriting in semi-structured data we need to take into account that the alphabet over which queries (the one we want to rewrite and the views to use in the rewriting) are expressed, is the set $\mathscr{F}$ of formulae of the underlying theory $\mathscr{T}$, and not the set of constants that appear as edge labels in graph databases.

In the following, let $Q_{0}$ be a regular path query and $\mathscr{Q}=\left\{Q_{1}, \ldots, Q_{k}\right\}$ be a finite set of views, also expressed as regular path queries, in terms of which we want to rewrite $Q_{0}$. Let $\mathscr{F}$ be the set of formulae of $\mathscr{T}$ appearing in $Q_{0}, Q_{1}, \ldots, Q_{k}$, and let $\mathscr{2}$ have an associated alphabet $\Sigma_{\mathscr{Q}}$ containing exactly one symbol for each view in $\mathscr{2}$. We denote the view associated with the symbol $q \in \Sigma_{\mathscr{Q}}$ with $\operatorname{rpq}(q)$.

Given a language $\ell$ over $\Sigma_{\mathscr{2}}$, we denote by $\exp _{\mathscr{F}}(\ell)$ the language over $\mathscr{F}$ defined as

$$
\exp _{\mathscr{F}}(\ell)=\bigcup_{q_{1} \cdots q_{n} \in \ell}\left\{w_{1} \cdots w_{n} \mid w_{i} \in L\left(r p q\left(q_{i}\right)\right)\right\}
$$

DEFINITION 4.3. Let $R$ be any formalism for defining a language $L(R)$ over $\Sigma_{2}$. $R$ is a rewriting of $Q_{0}$ wrt $\mathscr{Q}$ if for every database $D B$, ans $\left(\exp _{\mathscr{F}}(L(R)), D B\right)$ $\subseteq \operatorname{ans}\left(L\left(Q_{0}\right), D B\right)$, and is said to be

- maximal if for each rewriting $R^{\prime}$ of $Q_{0}$ wrt $\mathscr{Q}$ we have that $\operatorname{ans}\left(\exp _{\mathscr{F}}\left(L\left(R^{\prime}\right)\right)\right.$, $D B) \subseteq \operatorname{ans}\left(\exp _{\mathscr{F}}(L(R)), D B\right)$,

- exact if $\operatorname{ans}\left(\exp _{\mathscr{F}}(L(R)), D B\right)=\operatorname{ans}\left(L\left(Q_{0}\right), D B\right)$.

THEOREM 4.1. $R$ is a rewriting of $Q_{0}$ wrt $\mathscr{Q}$ if and only if $\operatorname{match}\left(\exp _{\mathscr{F}}(L(R))\right)$ $\subseteq \operatorname{match}\left(L\left(Q_{0}\right)\right)$. Moreover, $R$ is maximal if and only if for each rewriting $R^{\prime}$ of $Q_{0}$ wrt $\mathscr{Q}$ we have that match $\left(\exp _{\mathscr{F}}\left(L\left(R^{\prime}\right)\right)\right) \subseteq$ match $\left(\exp _{\mathscr{F}}(L(R))\right)$, and it is exact if and only if match $\left(\exp _{\mathscr{F}}(L(R))\right)=\operatorname{match}\left(L\left(Q_{0}\right)\right)$.

Proof. We prove only that $R$ is a rewriting of $Q_{0}$ wrt $\mathscr{Q}$ iff $\operatorname{match}\left(\exp _{\mathscr{F}}(L(R))\right.$ $\subseteq \operatorname{match}\left(L\left(Q_{0}\right)\right)$. The other assertions can be proved in a similar way.

" $\Rightarrow$ " By contradiction. Assume there exists a $\mathscr{D}$-word $a_{1} \cdots a_{n} \in \operatorname{match}\left(\exp _{\Sigma}\right.$ $[\mathscr{F}](L(R)))$ such that $a_{1} \cdots a_{n} \notin \operatorname{match}\left(L\left(Q_{0}\right)\right)$. Then for the database $D B$ consisting of a single path $x \stackrel{a_{1}}{\longrightarrow} \cdots \stackrel{a_{n}}{\longrightarrow} y$ it holds that $(x, y) \in \operatorname{ans}\left(\exp _{\mathscr{F}}(L(R))\right.$, $D B)$ but $(x, y) \notin \operatorname{ans}\left(L\left(Q_{0}\right), D B\right)$. Contradiction.

" $\Leftarrow "$ Again by contradiction. Assume there exists a database $D B$ and two nodes $x$ and $y$ in $D B$ such that $(x, y) \in \operatorname{ans}\left(\exp _{\mathscr{F}}(L(R)), D B\right)$ and $(x, y) \notin$ ans $\left(L\left(Q_{0}\right), D B\right)$. Then there exists a path $x \stackrel{a_{1}}{\longrightarrow} \cdots \stackrel{a_{n}}{\longrightarrow} y$ in $D B$ such that $a_{1} \cdots a_{n} \in \operatorname{match}\left(\exp _{\mathscr{F}}(L(R))\right)$. Hence $a_{1} \cdots a_{n} \in \operatorname{match}\left(L\left(Q_{0}\right)\right)$ and thus $(x, y) \in$ $\operatorname{ans}\left(L\left(Q_{0}\right), D B\right)$. Contradiction. 


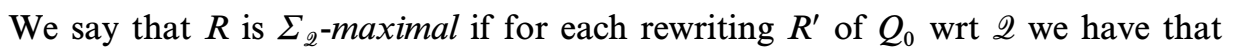
$L\left(R^{\prime}\right) \subseteq L(R)$. By arguing as in Theorem 2.1, and exploiting Theorem 4.1, it is easy to show that a $\Sigma_{2}$-maximal rewriting is also maximal.

Next we show how to compute a $\Sigma_{\mathscr{2}}$-maximal rewriting, by exploiting the construction presented in Section 2. Applying the construction literally, considering $\mathscr{F}$ as the base alphabet $\Sigma$, we would not take into account the theory $\mathscr{T}$, and hence the construction would not give us the maximal rewriting in general. As an example, suppose that $\mathscr{T} \vDash \forall x . A(x) \supset B(x), Q_{0}=B$, and $\mathscr{Q}=\{A\}$. Then the maximal rewriting of $Q_{0}$ wrt $\mathscr{Q}$ is $A$, but the algorithm would give us the empty language.

In order to take the theory into account, we proceed as follows: For each query $Q \in\left\{Q_{0}\right\} \cup \mathscr{Q}$ we construct an automaton $Q^{g}$ accepting the language match $(L(Q))$. This can be done by viewing the query $Q$ as a (possibly nondeterministic) automaton $Q=\left(\mathscr{F}, S, s_{0}, \rho, F\right)$ and constructing $Q^{g}$ as $\left(\mathscr{D}, S, s_{0}, \rho^{g}, F\right)$, where $s_{j} \in \rho^{g}\left(s_{i}, a\right)$ if and only if $s_{j} \in \rho\left(s_{i}, \varphi\right)$ and $\mathscr{T} \vDash \varphi(a)$. Observe that the set of states of $Q$ and $Q^{g}$

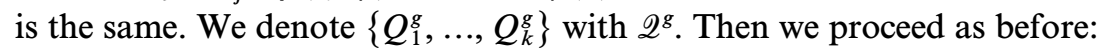

1. Construct a deterministic automaton $A_{d}=\left(\mathscr{D}, S_{d}, s_{0}, \rho_{d}^{g}, F_{d}\right)$ such that $L\left(A_{d}\right)=L\left(Q_{0}^{g}\right)$.

2. Define the automaton $A^{\prime}=\left(\Sigma_{2}, S_{d}, s_{0}, \rho^{\prime}, S_{d}-F_{d}\right)$, where $s_{j} \in \rho^{\prime}\left(s_{i}, q\right)$ if and only if there exists a word $w \in \operatorname{match}(L(r p q(q)))$, i.e., $w \in L\left(Q^{g}\right)$ where $Q=\operatorname{rpq}(q)$, such that $s_{j} \in \rho_{d}^{g *}\left(s_{i}, w\right)$.

3. Return $R_{\mathscr{Q}, Q_{0}}=R_{\mathscr{Q}^{g}, Q_{0}^{g}}=\overline{A^{\prime}}$.

THEOREM 4.2. The automaton $R_{\mathscr{Q}, Q_{0}}$ is a $\Sigma_{\mathscr{Q}}$-maximal rewriting of $Q_{0}$ wrt $\mathscr{Q}$.

Proof. First we show that every rewriting $R$ of $Q_{0}^{g}$ wrt $\mathscr{Q}^{g}$ is also a rewriting of $Q_{0}$ wrt $\mathscr{Q}$, and vice-versa. If $R$ is a rewriting of $Q_{0}^{g}$ wrt $\mathscr{2}^{g}$, then by definition $\exp _{\mathscr{D}}\left(L(R) \subseteq L\left(Q_{0}^{g}\right)\right.$, which implies that match $\left(\exp _{\mathscr{F}}(L(R))\right) \subseteq \operatorname{match}\left(L\left(Q_{0}\right)\right)$. Hence, by Theorem 4.1, $R$ is a rewriting of $Q_{0}$ wrt 2 . On the converse, if $R$ is a rewriting of $Q_{0}$ wrt $\mathscr{2}$, then by Theorem 4.1 match $\left(\exp _{\mathscr{F}}(L(R))\right) \subseteq$ match $\left(L\left(Q_{0}\right)\right)$, which implies that $\exp _{\mathscr{D}}\left(L(R) \subseteq L\left(Q_{0}^{g}\right)\right.$, i.e., $R$ is a rewriting of $Q_{0}^{g}$ wrt $\mathscr{Q}^{g}$.

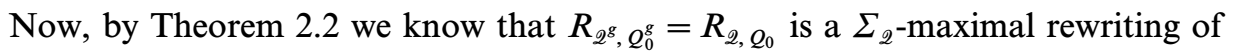

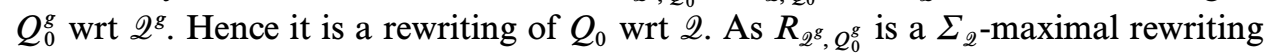
of $Q_{0}^{g}$ wrt $\mathscr{Q}^{g}$, we have that, for each rewriting $R$ of $Q_{0}^{g}$ wrt $\mathscr{2}^{g}$, and hence for each rewriting $R$ of $Q_{0}$ wrt $\mathscr{Q}, L(R) \subseteq L\left(R_{\mathscr{Q}^{g}}, Q_{0}^{g}\right)=L\left(R_{\mathscr{Q}, Q_{0}}\right)$, which implies that $R_{\mathscr{Q}, Q_{0}}$ is a $\Sigma_{\mathscr{Q}}$-maximal rewriting of $Q_{0}$ wrt $\mathscr{Q}$.

To check that $R_{\mathscr{Q}, Q_{0}}$ is an exact rewriting of $Q_{0}$ wrt $\mathscr{Q}$ we can proceed as in Section 2 , by constructing an automaton $B$ that accepts $\exp _{\mathscr{D}}\left(L\left(R_{\mathscr{Q}^{g}}, Q_{0}^{g}\right)\right)$, and checking for the emptiness of $L\left(A_{d} \cap \bar{B}\right)$.

Observe that both the size of $Q_{0}^{g}$ and $\mathscr{2}^{g}$ and the time needed to construct them from $Q_{0}$ and $\mathscr{Q}$ are linearly related to the size of $Q_{0}$ and $\mathscr{2}$. It follows that the same upper bounds as established in Section 3.1 hold for the case of regular path queries 5 .

\footnotetext{
${ }^{5} \mathrm{We}$ remind the reader that the number of constants in $\mathscr{D}$ is assumed to be constant.
} 
In fact, the construction of $\mathscr{2}^{g}$ can be avoided in building $R_{\mathscr{Q}, Q_{0}}$, since we can verify whether there exists a $\mathscr{D}$-word $w \in \operatorname{match}(L(r p q(q)))$ such that $s_{j} \in$ $\rho_{d}^{g *}\left(s_{i}, w\right)$ (required in Step 2 of the algorithm above) as follows. We consider directly the automaton $Q=r p q(q)$ (which is over the alphabet $\mathscr{F}$ ) and the automaton $A_{d}^{i, j}=\left(\mathscr{D}, S_{d}, s_{i}, \rho_{d}^{g},\left\{s_{j}\right\}\right)$ obtained from $A_{d}$ by suitably changing the initial and final states. Then we construct from $Q$ and $A_{d}^{i, j}$ the product automaton $K$, with the proviso that $K$ has a transition from $\left(s_{1}, s_{2}\right)$ to $\left(s_{1}^{\prime}, s_{2}^{\prime}\right)$ (whose label is irrelevant) if and only if (i) there is a transition from $s_{1}$ to $s_{1}^{\prime}$ labeled $a$ in $Q_{i, j}$, (ii) there is a transition from $s_{2}$ to $s_{2}^{\prime}$ labeled $\varphi$ in $Q$, and (iii) $\mathscr{T} \vDash \varphi(a)$. Finally, we check whether $K$ accepts a non-empty language. This allows us to instantiate the formulae in $\mathscr{Q}$ only to those constants that are actually necessary to generate the transition function of $A^{\prime}$.

With regard to $Q_{0}$, instead of constructing $Q_{0}^{g}$, we can build an automaton based on the idea of separating constants into suitable equivalence classes according to the formulae in the query they satisfy. The resulting automaton still describes the query $Q_{0}$, and its alphabet is generally much smaller than that of $Q_{0}^{g}$.

\subsection{Properties of Rewritings}

In the case where the rewriting $R_{\mathscr{Q}, Q_{0}}$ is not exact, the only thing we know is that such rewriting is the best one we can obtain by using only the views in 2 . However, one may want to know how to get an exact rewriting by adding to 2 suitable views.

EXAMPLE 4.1. Let $Q_{0}=a \cdot(b+c), \mathscr{Q}=\{a, b\}$, and $\Sigma_{\mathscr{Q}}=\left\{q_{1}, q_{2}\right\}$, where $r p q\left(q_{1}\right)$ $=a$, and $r p q\left(q_{2}\right)=b$. Then $R_{2, Q_{0}}=q_{1} \cdot q_{2}$, which is not exact. On the other hand, by adding $c$ to $\mathscr{2}$ and $q_{3}$ to $\Sigma_{2}$, with $\operatorname{rpq}\left(q_{3}\right)=c$, we obtain $q_{1} \cdot\left(q_{2}+q_{3}\right)$ as an exact rewriting of $Q_{0}$.

As an example, we consider the case where the views added to $\mathscr{Q}$ are atomic, i.e., have the form $\lambda z . P(z)$, where $P$ is a predicate of $\mathscr{T}$. Notice that atomic views include views of the form $\lambda z . z=a$, which we call elementary. The intuitive idea is to choose a subset $\mathscr{P}^{\prime}$ of the set $\mathscr{P}$ of predicates of $\mathscr{T}$, and to construct an exact rewriting of $Q_{0}$ wrt $\mathscr{Q}_{+}$, where $\mathscr{Q}_{+}$is obtained by adding to $\mathscr{Q}$ an atomic view for each symbol in $\mathscr{P}^{\prime}$. An exact rewriting $R$ of $Q_{0}$ wrt $\mathscr{Q}_{+}$is called a partial rewriting of $Q_{0}$ wrt $\mathscr{Q}$, provided that $\mathscr{Q}_{+} \neq \mathscr{Q}$.

The method we have presented can be easily adapted to compute partial rewritings. Indeed, if we compute $R_{\mathscr{Q}_{+}, Q_{0}}$, we obtain a partial rewriting of $Q_{0}$ wrt $\mathscr{2}$, provided that $R_{\mathscr{Q}_{+}, Q_{0}}$ is an exact rewriting of $Q_{0}$ wrt $\mathscr{Q}_{+}$. Observe that it is always possible to choose a subset $\mathscr{P}^{\prime}$ of $\mathscr{P}$ in such a way that $R_{\mathscr{Q}_{+}, Q_{0}}$ is exact (e.g., by choosing the set of all elementary views).

Typically, one is interested in using as few symbols of $\mathscr{P}$ as possible to form $\mathscr{Q}_{+}$, and this corresponds to choosing the minimal subsets $\mathscr{P}^{\prime}$ such that $R_{\mathscr{Q}_{+}, Q_{0}}$ is exact. More generally, one can establish various preference criteria for choosing rewritings. For instance, we may say that a (partial) rewriting $R$ is preferable to a (partial) rewriting $R^{\prime}$ if one of the following holds:

1. $\operatorname{match}\left(\exp _{\mathscr{F}}\left(L\left(R^{\prime}\right)\right)\right) \subset \operatorname{match}\left(\exp _{\mathscr{F}}(L(R))\right)$,

2. $\operatorname{match}(L(R))=\operatorname{match}\left(L\left(R^{\prime}\right)\right)$ and $R$ uses less additional atomic views than $R^{\prime}$, 
3. $\operatorname{match}(L(R))=\operatorname{match}\left(L\left(R^{\prime}\right)\right), \quad R$ uses the same number of additional atomic views as $R^{\prime}$, and less additional atomic nonelementary views.

4. $\operatorname{match}(L(R))=\operatorname{match}\left(L\left(R^{\prime}\right)\right), \quad R$ uses the same number of additional atomic and of additional nonelementary views as $R^{\prime}$, and less views than $R^{\prime}$.

Under this definition an exact rewriting is preferable to a nonexact one. Moreover, the definition reflects the fact that the cost of materializing additional atomic views (in particular the nonelementary ones) is higher than the cost of using the available ones. Finally, since a certain cost is associated with the use of each view, when comparing two rewritings defining the same language and using (if any) the same number of additional atomic and nonelementary views, then the one that uses less views is preferable.

The rewriting algorithm presented above can be immediately exploited to compute the most preferable rewritings according to the above criteria. It is easy to see that the problem of computing the most preferable rewritings remains in the same complexity class, since the complexity is dominated by the cost of computing the rewriting.

\section{CONCLUSIONS}

In this paper we have studied the problem of view-based query rewriting in the case where both the query and the views are expressed as regular path queries. We have shown the decidability of the problem of computing the maximal rewriting and checking whether it is exact. We have characterized the computational complexity of the problem and have provided algorithms that are essentially optimal. We envision several directions for extending the present work.

First, in this paper we focused on the problem of computing the maximal contained rewriting, i.e., the best rewriting that is guaranteed to provide only answers contained in those of the original query. Also of interest is the dual approach, i.e., computing the minimal containing rewritings (in general not unique), which guarantee to provide all the answers of the original query, and possibly more.

Second, we are interested in studying rewritings for more general forms of queries, such as the so-called generalized path queries, i.e., queries of the form $x_{1} Q_{1} x_{2} \cdots x_{n-1} Q_{n-1} x_{n}$, where each $Q_{i}$ is a regular path query [FS98]. Such queries ask for all $n$-tuples $o_{1}, \ldots, o_{n}$ of nodes such that, for each $i$, there is a path from $o_{i}$ to $o_{i+1}$ that satisfies $Q_{i}$. Notice that, since such queries compute $n$-ary relations (not necessarily binary ones), it is a priori not obvious in which language to express the rewriting. If one wants to use the operators for regular expressions in the rewriting, at least a projection operator that projects the $n$-ary relation on two of its components is needed. A further generalization would be to consider conjunctions of regular path queries, where the context in which a certain subpath appears is even more complex [CDGLV00b].

Third, one can investigate possible interesting subcases where the rewriting of regular (and generalized) path queries can be done more efficiently. Additionally, cost models for path queries and preference criteria that take into account such cost 
models can be defined, leading to the development of techniques for choosing the best rewriting with respect to the new criteria.

Finally, it is interesting to investigate the relationship between view-based query rewriting and view-based query answering in semi-structured data. The problem of view-based query answering is the one of computing the answer to a query having only information about the extensions of a set of materialized views [AD98]. Query rewriting techniques can in principle be used for view-based query answering. However, the precise relationship between the two problems is rather involved, as discussed in [CDGLV00a, CDGLV00c, CDGLV00d].

\section{ACKNOWLEDGMENTS}

This work was supported in part by the NSF Grants CCR-9628400, CCR-9700061, and IIS-9908435, by MURST, by ESPRIT LTR Project 22469 DWQ (Foundations of Data Warehouse Quality), and by the Italian Space Agency (ASI) under Project "Integrazione ed Accesso a Basi di Dati Eterogenee". Part of this work was done when the last author was a Varon Visiting Professor at the Weizmann Institute of Science.

\section{REFERENCES}

[Abi97] S. Abiteboul, Querying semi-structured data, in "Proc. of the 6th Int. Conf. on Database Theory (ICDT'97)," pp. 1-18, 1997.

[ACPS96] S. Adali, K. S. Candan, Y. Papakonstantinou, and V. S. Subrahmanian, Query caching and optimization in distributed mediator systems, in "Proc. of the ACM SIGMOD Int. Conf. on Management of Data," pp. 137-148, 1996.

[AD98] S. Abiteboul and O. Duschka, Complexity of answering queries using materialized views, in "Proc. of the 17th ACM SIGACT SIGMOD SIGART Symp. on Principles of Database Systems (PODS'98)," pp. 254-265, 1998.

[AGK99] F. N. Afrati, M. Gergatsoulis, and T. Kavalieros, Answering queries using materialized views with disjunction, in "Proc. of the 7th Int. Conf. on Database Theory (ICDT'99)," Lecture Notes in Computer Science, Vol. 1540, pp. 435-452. Springer-Verlag, Berlin, 1999.

[AQM+97] S. Abiteboul, D. Quass, J. McHugh, J. Widom, and J. L. Wiener, The Lorel query language for semistructured data, Int. J. Digital Libraries 1 (1997), 68-88.

[AV97] S. Abiteboul and V. Vianu, Regular path queries with constraints, in "Proc. of the 16th ACM SIGACT SIGMOD SIGART Symp. on Principles of Database Systems (PODS'97)," pp. 122-133, 1997.

[BDFS97] P. Buneman, S. Davidson, M. F. Fernandez, and D. Suciu, Adding structure to unstructured data, in "Proc. of the 6th Int. Conf. on Database Theory (ICDT'97)," pp. 336-350, 1997.

[BDHS96] P. Buneman, S. Davidson, G. Hillebrand, and D. Suciu, A query language and optimization technique for unstructured data, in "Proc. of the ACM SIGMOD Int. Conf. on Management of Data," pp. 505-516, 1996.

[Ber66] R. Berger, The undecidability of the dominoe problem, Mem. Amer. Math. Soc. 66 (1966), 1-72.

[BFW98] P. Buneman, W. Fan, and S. Weinstein, Path constraints on semistructured and structured data, in "Proc. of the 17th ACM SIGACT SIGMOD SIGART Symp. on Principles of Database Systems (PODS’98),” pp. 129-138, 1998. 
[BLR97] C. Beeri, A. Y. Levy, and M.-C. Rousset, Rewriting queries using views in description logics, in "Proc. of the 16th ACM SIGACT SIGMOD SIGART Symp. on Principles of Database Systems (PODS'97)," pp. 99-108, 1997.

[Bun97] P. Buneman, Semistructured data, in "Proc. of the 16th ACM SIGACT SIGMOD SIGART Symp. on Principles of Database Systems (PODS'97)," pp. 117-121, 1997.

[CACS94] V. Christophides, S. Abiteboul, S. Cluet, and M. Scholl, From structured documents to novel query facilities, in "Proc. of the ACM SIGMOD Int. Conf. on Management of Data” (R. T. Snodgrass and M. Winslett, Eds.), Minneapolis, MI, pp. 313-324, 1994.

[CDGL98] D. Calvanese, G. De Giacomo, and M. Lenzerini, On the decidability of query containment under constraints, in "Proc. of the 17th ACM SIGACT SIGMOD SIGART Symp. on Principles of Database Systems (PODS'98)," pp. 149-158, 1998.

[CDGLV00a] D. Calvanese, G. De Giacomo, M. Lenzerini, and M. Y. Vardi, Answering regular path queries using views, in "Proc. of the 16th IEEE Int. Conf. on Data Engineering (ICDE 2000)," pp. 389-398, 2000.

[CDGLV00b] D. Calvanese, G. De Giacomo, M. Lenzerini, and M. Y. Vardi, Containment of conjunctive regular path queries with inverse, in "Proc. of the 7th Int. Conf. on Principles of Knowledge Representation and Reasoning (KR'2000)," pp. 176-185, 2000.

[CDGLV00c] D. Calvanese, G. De Giacomo, M. Lenzerini, and M. Y. Vardi, Query processing using views for regular path queries with inverse, in "Proc. of the 19th ACM SIGACT SIGMOD SIGART Symp. on Principles of Database Systems (PODS 2000),” pp. 58-66, 2000.

[CDGLV00d] D. Calvanese, G. De Giacomo, M. Lenzerini, and M. Y. Vardi, View-based query processing and constraint satisfaction, in "Proc. of the 15th IEEE Symp. on Logic in Computer Science (LICS 2000)," pp. 361-371, 2000.

[CKPS95] S. Chaudhuri, S. Krishnamurthy, S. Potarnianos, and K. Shim, Optimizing queries with materialized views, in "Proc. of the 11th IEEE Int. Conf. on Data Engineering (ICDE’95)," Taipei (Taiwan), 1995.

[CM90] M. P. Consens and A. O. Mendelzon, Graphlog: a visual formalism for real life recursion, in "Proc. of the 9th ACM SIGACT SIGMOD SIGART Symp. on Principles of Database Systems (PODS’90)," Atlantic City, NJ, pp. 404 416, 1990.

[CMW87] I. F. Cruz, A. O. Mendelzon, and P. T. Wood, A graphical query language supporting recursion, in "Proc. of the ACM SIGMOD Int. Conf. on Management of Data," San Francisco, CA, pp. 323-330, 1987.

[CNS99] S. Cohen, W. Nutt, and A. Serebrenik, Rewriting aggregate queries using views, in "Proc. of the 18th ACM SIGACT SIGMOD SIGART Symp. on Principles of Database Systems (PODS'99)," pp. 155-166, 1999.

[Con71] J. H. Conway, "Regular Algebra and Finite Machines," Chapman and Hall, London, 1971.

[DG97] O. M. Duschka and M. R. Genesereth, Answering recursive queries using views, in "Proc. of the 16th ACM SIGACT SIGMOD SIGART Symp. on Principles of Database Systems (PODS'97)," pp. 109-116, 1997.

[DG98] O. M. Duschka and M. R. Genesereth, Query planning with disjunctive sources, in "Proc. of the AAAI-98 Workshop on AI and Information Integration," AAAI Press/ The MIT Press, Cambridge, MA, 1998.

[DGL00] O. M. Duschka, M. R. Genesereth, and A. Y. Levy, Recursive query plans for data integration, J. Logic Program. 43 (2000), 49-73.

[FFK+98] M. F. Fernandez, D. Florescu, J. Kang, A. Y. Levy, and D. Suciu, Catching the boat with strudel: Experiences with a web-site management system, in "Proc. of the ACM SIGMOD Int. Conf. on Management of Data," pp. 414 425, 1998.

[FFLS97] M. F. Fernandez, D. Florescu, A. Y. Levy, and D. Suciu, A query language for a web-site management system, SIGMOD Record 26 (1997), 4-11. 
[FLS98] D. Florescu, A. Levy, and D. Suciu, Query containment for conjunctive queries with regular expressions, in "Proc. of the 17th ACM SIGACT SIGMOD SIGART Symp. on Principles of Database Systems (PODS'98),” pp. 139-148, 1998.

[FS98] M. F. Fernandez and D. Suciu, Optimizing regular path expressions using graph schemas, in "Proc. of the 14th IEEE Int. Conf. on Data Engineering (ICDE'98)," pp. 14-23, 1998.

[Hal00] A. Y. Halevy, Theory of answering queries using views, SIGMOD Record 29 (2000), 40-47.

[HU79] J. E. Hopcroft and J. D. Ullman, "Introduction to Automata Theory, Languages, and Computation," Addison-Wesley, Reading, MA, 1979.

[Jon75] N. D. Jones, Space-bounded reducibility among combinatorial problems, J. of Computer and System Sciences 11 (1975), 68-75.

[LMSS95] A. Y. Levy, A. O. Mendelzon, Y. Sagiv, and D. Srivastava, Answering queries using views, in "Proc. of the 14th ACM SIGACT SIGMOD SIGART Symp. on Principles of Database Systems (PODS'95)," pp. 95-104, 1995.

[MMM97] A. Mendelzon, G. A. Mihaila, and T. Milo, Querying the World Wide Web, Int. J. on Digital Libraries 1 (1997), 54-67.

[MS99] T. Milo and D. Suciu, Index structures for path expressions, in "Proc. of the 7th Int. Conf. on Database Theory (ICDT'99)," Lecture Notes in Computer Science, Vol. 1540, pp. 277-295, Springer-Verlag, Berlin, 1999.

[PV99] Y. Papakonstantinou and V. Vassalos, Query rewriting using semistructured views, in "Proc. of the ACM SIGMOD Int. Conf. on Management of Data," 1999.

[QRS +95] D. Quass, A. Rajaraman, I. Sagiv, J. Ullman, and J. Widom, Querying semistructured heterogeneous information, in "Proc. of the 4th Int. Conf. on Deductive and ObjectOriented Databases (DOOD'95)," pp. 319-344, Springer-Verlag, Berlin, 1995.

[RS59] M. O. Rabin and D. Scott, Finite automata and their decision problems, IBM J. Res. Develop. 3 (1959), 115-125.

[RSU95] A. Rajaraman, Y. Sagiv, and J. D. Ullman, Answering queries using templates with binding patterns, in "Proc. of the 14th ACM SIGACT SIGMOD SIGART Symp. on Principles of Database Systems (PODS'95)," 1995.

[Sav70] W. J. Savitch, Relationship between nondeterministic and deterministic tape complexities, J. Comp. System Sci. 4 (1970), 177-192.

[SDJL96] D. Srivastava, S. Dar, H. V. Jagadish, and A. Levy, Answering queries with aggregation using views, in "Proc. of the 22nd Int. Conf. on Very Large Data Bases (VLDB'96)," pp. 318-329, 1996.

[TSI96] O. G. Tsatalos, M. H. Solomon, and Y. E. Ioannidis, The GMAP: A versatile tool for phyisical data independence, Very Large Database J. 5 (1996), 101-118.

[U1197] J. D. Ullman, Information integration using logical views, in "Proc. of the 6th Int. Conf. on Database Theory (ICDT'97)," Lecture Notes in Computer Science, Vol. 1186, pp. 19-40, Springer-Verlag, Berlin, 1997.

[vEB82] P. van Emde Boas, Dominoes are forever, in "Proc. of 1st GTI Workshop, Rheie Theoretische Informatik UGH Paderborn,” pp. 75-95, Paderborn, Germany, 1982.

[vEB97] P. van Emde Boas, The convenience of tilings, in "Complexity, Logic, and Recursion Theory" (A. Sorbi, Ed.), Lecture Notes in Pure and Applied Mathematics, Vol. 187, pp. 331-363, Marcel Dekker, 1997. 